\title{
Making the Most of Youth Mentoring: A Guide for Funders
}

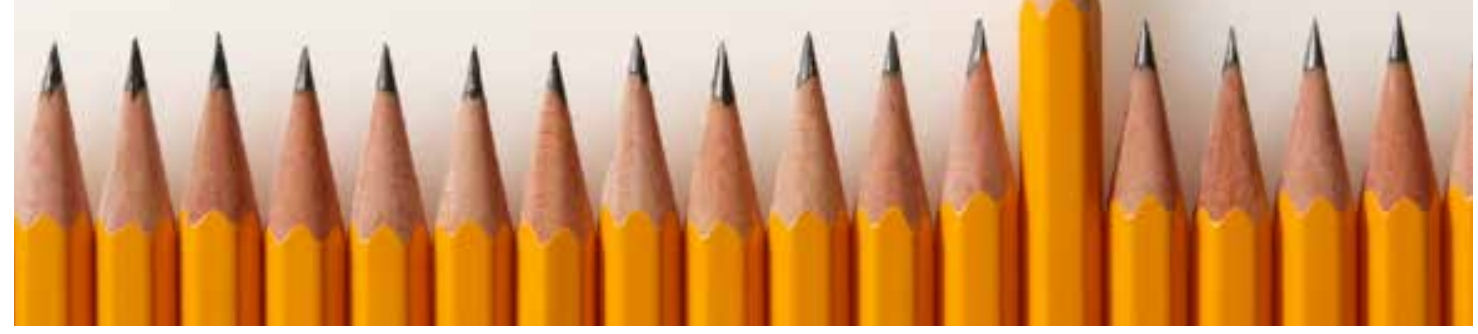

Carla Herrera

The mentoring field has grown tremendously over the last two decades. There are now thousands of mentoring programs around the country, and many initiatives that incorporate elements of mentoring into larger sets of services. Funders and policymakers who want to improve youth outcomes are faced with tough decisions about how to allocate their scarce resources in a crowded and complex field.

Mentoring has a rich research base, but a large variety of program models, including community-based, school-based, group, email, peer and paid mentoring approaches. Research across these programs is uneven: Some approaches have been studied rigorously at scale, while others have very limited evidence of effectiveness. The research that does exist suggests that these programs are not always effective with the same groups of youth, nor can they be expected to achieve the same goals. Each approach may require its own set of resources and capacities, with different "best practices" needed to yield benefits.

Funders and program leaders are left with a dizzying array of mentoring approaches to choose from and very little guidance about which one may be the best fit for the youth they hope to reach.

In this guide:

1. Tips for recognizing organizations with the capacity to implement strong mentoring programs.

2. Tips for recognizing high-quality mentoring programs.

3. Tips for making the most of different mentoring approaches:

- Community-based mentoring

- School-based mentoring

- Group or team mentoring

- Cross-age peer mentoring

- E-mentoring

- Paid mentoring 
To address this need, The Pinkerton Foundation asked Public/Private Ventures (P/PV) to develop a brief guide, outlining which types of mentoring programs work, for whom and under what circumstances. To achieve this, we mined our own 25-plus years of work in mentoring; reviewed key literature; and gathered input from leading experts in the field: Michael Garringer (National Mentoring Center, Education Northwest), Michael Karcher (University of Texas at San Antonio), and Jean Rhodes (University of Massachusetts, Boston). ${ }^{1}$ Our goal was to help funders invest their limited resources where they can have the most impact by: (1) determining which organizations have the capacity to implement strong mentoring programs; (2) identifying the features that undergird high-quality mentoring programs; and (3) understanding how to make the most of distinct mentoring approaches (including best practices, and red flags to avoid, for each type of mentoring reviewed).

This guide is by no means conclusive, but it should help funders navigate the existing research on mentoring and provide a starting framework for deciding which programs to support.

\section{Recognizing Organizations with the Capacity 1 to Implement Strong Mentoring Programs}

As with all social programs, the stability and strength of a mentoring program is determined at least in part by the character of the organization implementing it. There are a number of core organizational features that are crucial to ensure that a program is well managed and financially stable. These broad organizational features are examined in more detail in other work, and we include them here essentially as a reminder. When considering funding for an organization that provides mentoring services, grantmakers should start by asking 10 basic sets of questions:

Does the organization have an institutional history or connection to mentoring (i.e., expertise in mentoring or connection to an established mentoring program)? How central is mentoring to the organization's mission and core capacities?

Does the organization have a governing body (i.e., a board)? Is the board invested in the mentoring program? Does the board offer significant help with fundraising for the program?
Does the organization have partnerships (or plans for partnerships) that will help it recruit youth and volunteers for the mentoring program?

Is the staffing of the program adequate, with appropriate qualifications, roles, clinical expertise, etc.? Do staff have access to technical assistance, training and staff development opportunities that are applicable to the specific groups of youth with whom they are working? 
Has the organization/program had significant issues with staff turnover, particularly recent turnover in key management positions? Does it have a clear plan for how to retain its staff and deal with turnover?

$\square$ If you are funding growth or expansion in the program/organization, does the organization have the experience and capacity to manage this growth?

Does the organization have the experience, capacity and resources to manage finances effectively? $\square$ Does the organization have a diverse enough funding base that it could survive without your funding if needed?

$\square$ Does the organization have appropriate physical space to house its operations and, if applicable, activities for youth participants? Does it have computers, software, and other technology resources needed to manage the program and fundraise effectively?

What is the relationship of the organization to the community? How is it viewed by the community? Are there "competing" organizations offering similar services?

If an organization doesn't meet all of these criteria, that doesn't necessarily make it a bad investment. But the answers to these questions do present a clearer picture of an organization's strengths and weaknesses. Funders may need to decide if they are willing to make a larger investment in the organization's infrastructure (versus just funding the program under consideration) to lay a solid foundation for success.

\section{O Recognizing High-Quality Mentoring Programs}

In addition to the "parent" organization's core features, strong mentoring programs also have specific characteristics that help them create high-quality, effective mentoring relationships. Fundamentally, these characteristics fall into two categories: the idea behind the program, and how that idea is implemented.

Regardless of the particular mentoring model, the following questions can go a long way toward helping funders determine if the program is a good investment. Lacking one or two of these features shouldn't be viewed as a "deal breaker"; however lacking several, within each of these two key areas, may be cause for concern.

\section{Is the idea behind the program well articulated and integrated with other program elements? (i.e., "Is it really mentoring?")}

Many mentoring programs have a broad theory of change, ${ }^{2}$ but fewer have a theory of change that truly guides their work with youth-determining program goals, activities and components in a clear and logical way that reflects current research. (See Figure 1 on the next page, for a sample theory of change depicting one route through which a school-based mentoring program might promote changes in youth's academic behavior 
and performance.) The following checklist will help ensure that a program's theory of change is appropriate for mentoring and that it is reflected in how the program serves youth on the ground.

Does the program have a stated goal to create relationships between mentors and youth (the program may be strong, but not necessarily a mentoring program, in which adult-youth relationships are central)?

$\square$ Is the mentoring relationship a key mechanism of change in the program?

$\square$ Is the program model-the set of activities and services delivered in the chosen setting and supported by auxiliary activities (such as training and support) - likely to result in the creation of a trusting relationship between the mentor and youth?

प Can program staff clearly articulate how participants' lives will be different while they are in the program and after their involvement (i.e., in addition to a long-term vision for improving youth's lives, does the program have short- and medium-term goals for individual youth that are realistic and logically connected to the experiences and services provided by the program)?

$\square$ Does the expected length of the mentoring "match" (i.e., the mentor's time commitment) align with the program's theory of change (e.g., in programs where the relationship itself is the only mechanism of change, does the program ask volunteers to commit enough time for a strong mentoring relationship to develop)?

Does the program have some way of assessing whether its goals are achieved?

$\square$ When mentoring is a component of larger systems or sets of services, is it logically and sensibly integrated into the overall service offerings, to support the short- and medium-term outcomes that are desired?

Figure 1:

\section{Sample Theory of Change for a School-Based Mentoring Program}

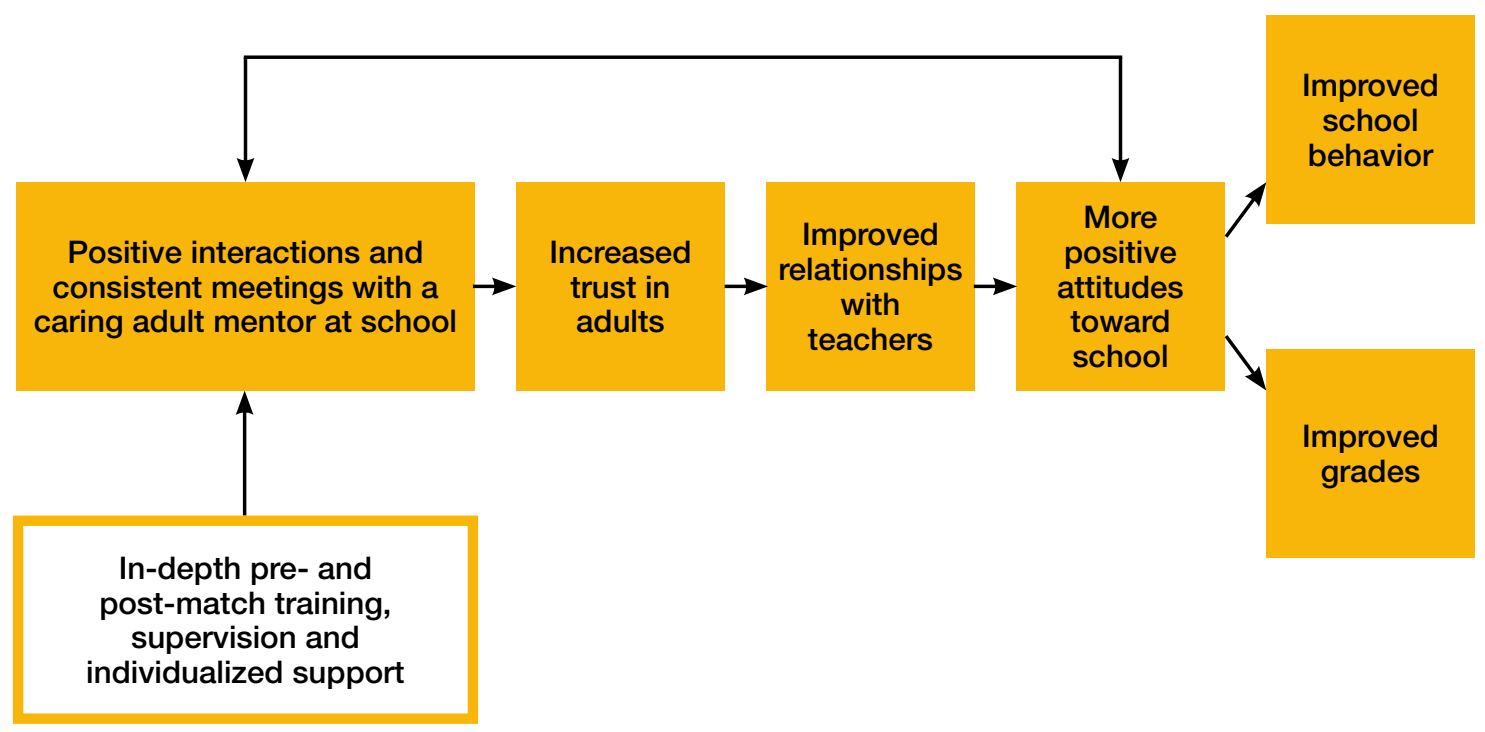




\section{Is the program designed around solid, research-based practices, and are these practices well implemented? (i.e., "Is it good mentoring?")}

Mentoring has a strong research base that links particular program features and practices with the creation of effective mentoring relationships and with a variety of specific outcomes. Of course, programs can easily point to "best practices" that they loosely follow (e.g., "required training" can range from a pamphlet offered at intake to a much more in-depth and productive hands-on experience for mentors). Thus, in addition to assessing whether a given practice is implemented, it is also important to assess how well that practice is implemented, and the extent to which program practices are actually experienced by mentors and families as helpful. Funders can ask a handful of more in-depth questions of program staff, examine a random sample of case notes or contact a small group of mentors or participating families to hear more about their experiences. The following questions should serve as a starting point for this kind of more in-depth exploration.

Does the program implement the Elements of Effective Practice for Mentoring ${ }^{3}$ (which reflect research about what works in mentoring programs), with clear benchmarks and guidelines for:

$\Leftrightarrow$ Recruiting youth and mentors;

$\Leftrightarrow$ Screening youth and mentors;

$\Leftrightarrow$ Procedures for matching youth with mentors;

$\Leftrightarrow$ Targeted length of match and frequency of contact between mentors and youth;

$\Leftrightarrow$ Frequency and content of supervision;

$\Leftrightarrow$ Length, content and timing of mentor training; and

$\Leftrightarrow$ Structured processes to end the match (e.g., a closure ritual for the match; an exit interview for the mentor and mentee separately; support for parents in how to manage match endings)?

Does the program offer tailored mentor support and training in response to specific circumstances and crises that may arise during the match and are relevant to the special needs of the youth being served (e.g., mentors of youth in foster care may need training on such topics as: working with changing guardianship, navigating the foster care system, and managing challenging behavior)? $\square$ Does the program have evidence that mentor and youth participants find the supports provided by the program helpful? For instance, can the agency provide evidence that mentors take advantage of trainings and/or that they find them useful? Can the agency provide evidence that mentors speak with case managers regularly and that these conversations are sufficient to meet their needs?

$\square$ Does the program have a youth-/matchtracking database that allows staff to examine basic information about the youth and mentors and the length of their matches?

Does the program regularly review data about its matches, youth and/or mentors? How does the program use that information to improve?

$\square$ Does the agency collect information about matches that close and why they close (i.e., does the agency actively try to understand what it could be doing to create stronger matches)?

What is the program's rate of early (i.e., prior to established program guidelines) match closures? Do most relationships (i.e., more than 50 percent) remain intact for the expected length of time? 


\section{Making the Most of Different Types of Mentoring Programs}

Different mentoring models often serve distinct groups of youth, involve different types of volunteers, and typically aim to achieve different goals. These models thus carry with them different indicators of strength and distinct "red flags" (i.e., practices whose presence or absence may indicate a weaker program). Six of the most common types of mentoring programs are outlined below. It should be noted that all mentoring programs, regardless of the particular model, should have the basic components listed in the previous section, including thorough background checks, training and regular, ongoing supervision for volunteers. The indicators presented here are in addition to those basics.

\section{COST}

\$ Least expensive.

\$ \$ Based on costs for community-based mentoring. Early estimates were about $\$ 1,000$ per youth per year; current estimates from practitioners are closer to $\$ 1,500$.

\$\$ Most expensive.

NOTE: Rigorous cost estimates do not exist for many mentoring approaches, and most that do are outdated. Thus, these categories are based on community-based mentoring costs (the "middle" category). The categories also have loose boundaries - programs can easily move up a category when using expensive curriculum, activities, expert mentors, extensive support, etc.

\section{EVIDENCE BASE}

Has no (or very few) rigorous published studies testing its effects and no (or very few) studies outlining strong practices.

OO Has some small-scale published studies testing its effects and a few studies outlining strong practices.

QO Has been tested rigorously in largescale studies, but in a limited number of programs.

QQO Has been tested rigorously at scale in several studies/contexts, with several studies assessing implementation.

NOTE: It is important to distinguish between "being rigorously tested" (rated here) versus "being effective." These scores do not reflect how effective a particular approach is, but rather how much research has been conducted on the approach to date, in part a function of age/prevalence of the approach.

\section{A COMMUNITY-BASED approach should be considered by funders who:}

WANT TO PROVIDE youth with:

$\Leftrightarrow 3$ to 4 hours a week of (typically) one-on-one, unstructured adult interaction.

$\Leftrightarrow$ An adult friend who can expose them to a range of experiences and provide help and advice on a wide and varying set of topics.

$\Leftrightarrow$ A program that can follow youth through residential moves.

\section{WANT TO SERVE youth:}

$\Leftrightarrow$ Across a wide range of ages.

$\Leftrightarrow$ Who are comfortable spending time alone with a non-familial adult. 
WANT TO INVOLVE adult mentors:

$\Leftrightarrow$ Who have access to reliable transportation.

$\Leftrightarrow$ Who have 3 to 4 hours a week to volunteer.

$\Leftrightarrow$ Who can commit to at least one year of regular meetings.

- WANT TO ACHIEVE:

$\Leftrightarrow$ A wide range of effects, including improving relationships with peers and adults, decreasing delinquent behavior, and improving youth's academic outcomes.

$\Leftrightarrow$ Outcomes that are not conducive to a "site-based" approach that is implemented in a specific location, such as a youth-serving organization, school or workplace (e.g., exposure to new experiences in and outside of youth's community).

$\Leftrightarrow$ Long-lasting mentor-youth relationships.

\section{Red flags for Community-Based programs:}

SCREENING: Program does not provide extensive screening and supervision of volunteers, including driver's license checks.

SUPERVISION: Program lacks a parent component in its match supervision, including a parent orientation; regular, ongoing communication with parents; and other strategies to ensure parental support of the match and child safety.

TRAINING: Program lacks in-person mentor training prior to the beginning of the match.

RECRUITMENT: Program primarily recruits mentors who may want or need more supervision or structure (e.g., youth mentors) than a community-based approach is typically structured to provide.

STRUCTURE: Program has very specific goals for youth (e.g., high school graduation) without providing mentors with related supports (e.g., structure, activity ideas and resources connected to those goals).

A SCHOOL-BASED approach should be considered by funder's who:

- WANT TO PROVIDE youth with:

$\Leftrightarrow$ A group or one-on-one mentoring experience (structured or unstructured) that occurs in a context where there are adults to supervise and resources to support the match.

$\Leftrightarrow$ About one hour a week of academic or social activities that are conducive to the school setting and calendar (i.e., during the school year).

\section{- WANT TO SERVE:}

$\Leftrightarrow$ Younger youth with school-related needs who are typically referred by their teachers.

$\Leftrightarrow$ Youth whose parents may not seek out a community-based mentoring program. 


\section{WANT TO INVOLVE:}

$\Leftrightarrow$ Young or elder mentors with limited transportation.

$\Leftrightarrow$ Volunteers who may need or want additional structure or supervision.

$\Leftrightarrow$ Volunteers who want a more time-limited experience.

$\Leftrightarrow$ Large groups of volunteers through a business or school (e.g., college- or high-schoolaged mentors).

\section{WANT TO ACHIEVE:}

$\Leftrightarrow$ Benefits primarily in school-related attitudes, behaviors and experiences, including improvements in peer relationships and academic attitudes, as well as decreases in truancy and misconduct at school.

\section{Red flags for School-Based programs:}

SCREENING: Program does not conduct background checks appropriate for involvement in the school setting.

\section{SUPERVISION:}

Program does not ensure regular, on-site supervision of match meetings.

4 Program does not provide frequent one-on-one check-ins with mentors and troubleshooting outside of match meetings.

TRAINING: Program lacks mentor training about how to work within the school context (e.g., how/whether to include youth's peers in interactions, how to work/interact with teachers and administrative staff).

STRUCTURE: Program serves high-school-aged mentees without modifying the model in ways that reflect this age group's specific needs.

\section{PARTNERS:}

4 School staff have no input into decisions about the program's implementation (e.g., when, how and where it takes place).

4 School staff have the only or primary input into decisions about the program's central goals and content, potentially pulling the program away from its central theory of change. Program has no mechanism for collecting information from school staff on how it could be improved and for responding to these suggestions.

4 Program lacks partnerships with supportive schools that are willing to invest resources (e.g., time, activities, computers, designated space for meetings) to help the matches succeed, or it lacks the capacity to create these partnerships.

STAFFING: The school does not provide a point person on site for mentors to turn to when problems arise. 


\section{A GROUP or TEAM MENTORING approach should be considered by funders who:}

- WANT TO PROVIDE youth with:

$\Leftrightarrow$ A structured, group-based experience in which youth can interact with peers and one or more adults.

$\Leftrightarrow$ A (typically) site-based approach with a consistent time and place for meetings.

- WANT TO SERVE youth:

$\Leftrightarrow$ Both in and beyond elementary school (particularly older youth who are attracted to opportunities for social interaction with peers).

$\Leftrightarrow$ Who may be uncomfortable alone with a non-familial adult.

- WANT TO INVOLVE adult mentors:

$\Leftrightarrow$ Who want a site-based approach with more structure.

$\Leftrightarrow$ Who have experience and/or extensive training in managing groups of youth.

- WANT TO ACHIEVE:

$\Leftrightarrow$ Social and behavioral goals for youth (e.g., developing social skills, providing increased opportunities for social interaction, improving problem behavior, etc.).

\section{Red flags for Group or Team Mentoring programs:}

SCREENING: Program does not implement rigorous screening, particularly when it includes unsupervised group/match meetings.

\section{SUPERVISION:}

4 Program does not implement group observation as part of the supervision process.

4 Program does not incorporate "checks" to ensure that peers aren't encouraging/modeling negative behavior.

TRAINING: Program does not provide volunteers with significant training in group dynamics and peer relationships.

POPULATION: Program exclusively or predominantly serves youth with aggression or other behavior problems (mixed groups with predominantly "lower-risk" youth will help avoid "contagion" effects in which youth adopt the negative behaviors of their peers).

\section{STRUCTURE:}

4 Program creates groups with a very high youth-to-mentor ratio or a ratio that does not "match" the goals it is trying to achieve or the types of youth it targets (e.g., youth with behavioral issues and groups with very individualized goals may need more focused adult attention).

Program does not provide structure, activities and/or curriculum to focus group interactions. 
- WANT TO PROVIDE:

$\Leftrightarrow$ Children with a supervised (typically school-based) opportunity to spend time with older peers and observe older youth modeling caring, supportive behavior.

$\Leftrightarrow$ Older youth with opportunities for leadership, volunteerism, community building and positive interactions with peers.

- WANT TO SERVE:

$\Leftrightarrow$ Younger youth, especially elementary-aged.

$\Leftrightarrow$ Possibly middle-school-aged youth (increasing numbers of cross-age peer mentoring programs involve middle school youth being mentored by high school students; these matches require more programmatic support to avoid older peers modeling, and mentees adopting, negative behaviors).

\section{- WANT TO INVOLVE:}

$\Leftrightarrow$ Large groups of high school student mentors.

$\Leftrightarrow$ High school volunteers who want the opportunity to develop relationships with younger youth, to serve as leaders, to give back to their community, or to test whether they might want to pursue a career working with children.

$\Leftrightarrow$ A cadre of teenage volunteers with the potential to improve the climate of their own high school, especially in schools where there may be limited opportunities for teens to engage in positive youth programming.

\section{WANT TO ACHIEVE:}

$\Leftrightarrow$ Improvements in older youth's (i.e., mentors') confidence in their academic abilities.

$\Leftrightarrow$ Improvements in mentees' relationships with peers and feelings of "connectedness" (i.e., feeling connected to school, parents and/or teachers).

$\Leftrightarrow$ Potential for later volunteering among the mentors.

$\Leftrightarrow$ Potential for improvements in school climate for both mentors and mentees.

\section{Red flags for Cross-Age Peer Mentoring programs:}

\section{SCREENING:}

4 Program does not have a mechanism to identify and screen out volunteers who are overcommitted or are volunteering solely to fulfill a course requirement.

4 Program recruits full classes of student mentors without screening each individual student before allowing them into the course.

SUPERVISION: Program does not provide in-person supervision for all match meetings (ideally additional staff or older peers help to lead meetings so that program staff can instead focus on carefully monitoring interactions among individual matches).

TRAINING: Program lacks extensive pre- and post-match training for volunteers (for teens, pre-match training alone is insufficient). 
RECRUITMENT: Program recruits full classes of student mentors when the classes are not specifically designed for mentoring.

\section{STRUCTURE:}

Program provides high school volunteers with course credit that isn't clearly tied to the program's guidelines (e.g., mentors receive credit before their time commitment is met).

4 Program does not provide structure for match interactions (without an evidence-based curricula with activities that can unite mentors and mentees, matches will likely flounder, mentors may congregate with their peers rather than spending time with their mentees, and mentees may adopt negative behaviors).

4 Program allows individual matches to meet in close proximity to other matches without structure and strategies to ensure that the mentor's attention is focused on his or her mentee, rather than on peers.

PARTNERS: Program lacks partnerships with supportive high schools to provide volunteers - schools that are willing to invest resources (e.g., a designated class period for attending mentoring sessions, transportation to the host school) to help the program succeed, or it lacks sufficient capacity to create these partnerships.

E-MENTORING

\section{should be considered by funder's who:}

- WANT TO PROVIDE youth with:

$\Leftrightarrow$ A structured mentoring experience.

$\Leftrightarrow$ A way to connect with a caring adult who may live far away.

$\Leftrightarrow$ An opportunity to write and/or learn to express feelings in writing.

$\Leftrightarrow$ Additional or intensive support with a project or specific area of study.

- WANT TO SERVE:

$\Leftrightarrow$ Older youth who have access to the internet and comfort and experience using email.

$\Leftrightarrow$ Youth who may prefer internet communication and/or have difficulty developing relationships through face-to-face interactions.

$\Leftrightarrow$ Youth who have special needs (e.g., youth with disabilities) that might prevent them from developing an in-person relationship with an adult mentor.

\section{WANT TO INVOLVE:}

$\Leftrightarrow$ Adult volunteers with access to the internet and comfort and experience using email.

$\Leftrightarrow$ Adults who have limited time and/or transportation to get to match meetings.

$\Leftrightarrow$ Large groups of volunteers through a business or school.

$\Leftrightarrow$ Volunteers who are isolated or have special needs (e.g., physical disabilities) that might prevent them from developing an in-person relationship with youth.

$\Leftrightarrow$ Volunteers who are far removed geographically from the youth they will serve or are difficult to meet with in person (e.g., celebrities). 
$\Leftrightarrow$ Volunteers who share a particular interest with their mentees or have a specific skill youth would like to develop (i.e., the match has a platform from which it can begin a relationship without the benefit of in-person discussions).

- WANT TO ACHIEVE:

$\Leftrightarrow$ Targeted outcomes (e.g., a specific academic skill or enhanced knowledge in a specific area), based on a curriculum or activities of focus.

$\Leftrightarrow$ Increased use and comfort with internet-based communication.

\section{Red flags for E-Mentoring programs:}

SCREENING: Program does not implement rigorous screening comparable to that in more traditional programs.

\section{SUPERVISION:}

Program does not have an online system that includes significant checks for frequency of email exchanges, email content and child safety.

4 Program does not provide supervision that is as frequent and intensive as that provided in more traditional mentoring programs.

TRAINING: Program does not train volunteers in how to communicate with youth online in ways that are most likely to foster meaningful, effective relationships.

\section{STRUCTURE:}

Program does not have curricula and/or clear goals and discussion topics/prompts to help focus match interactions.

4 Program does not include opportunities for social (i.e., non-task-focused) and face-to-face interactions, which may bolster program effects.

4 Program does not have outlined standards for timing, length and frequency of communication (i.e., at least weekly), including turnaround time for email responses.

\section{STAFFING:}

Site-based programs do not have a program manager at the site (e.g., school) where youth participate in program activities (i.e., youth have no in-person guidance and support during their key communications with mentors).

4 Program coordinators do not have experience supervising or working with people online.

\section{A PAID MENTORING approach}

\section{should be considered by funders who:}

\section{$\mathbf{\$ \$} \mid$ O}

\section{- WANT TO PROVIDE:}

$\Leftrightarrow$ A long-term and/or intensive mentoring experience in which mentors receive additional training and support to serve challenging youth, and/or whose level of involvement requires mentors to be paid for at least some of their work with youth.

$\Leftrightarrow$ An approach that ideally begins serving youth early (e.g., in the early years of school, as youth enter foster care, or before youth begin the reentry process). ${ }^{4}$ 
WANT TO SERVE:

$\Leftrightarrow$ Youth who need a long-term and/or intensive, stable mentoring relationship.

$\Leftrightarrow$ Youth who would benefit from a mentoring relationship that can stay with them through difficult transitions (e.g., from elementary to middle to high school, through residential moves in foster care, as part of reentry from institutional settings).

- WANT TO INVOLVE:

$\Leftrightarrow$ Mentors with special training or expertise (or who are willing to engage in intensive training) to serve the type of youth the program targets.

$\Leftrightarrow$ Mentors who are willing to commit to more time or more frequent, consistent meetings than volunteers in standard programs.

$\Leftrightarrow$ Mentors who can see, support and build on the positive attributes of the youth they work with (i.e., are able to see beyond youth's challenges).

- WANT TO ACHIEVE:

$\Leftrightarrow$ Long-term changes that require a more lengthy or intensive mentoring experience.

$\Leftrightarrow$ Improved outcomes for "higher-risk" youth, which are difficult to achieve in short-term programs (e.g., reduced recidivism, reduced drop-out rates, lower rates of teen pregnancy).

\section{Red flags for Paid Mentoring programs:}

\section{SCREENING:}

4 Program does not have mechanisms to screen out mentors who are motivated solely by payment.

4 Program does not have multiple, regular (e.g., annual) screenings when mentors are involved for more than one year.

\section{SUPERVISION:}

Program does not have a mechanism to detect and counteract mentor burnout (a real risk, given the significant time commitment required and the challenges faced by many of the youth in these programs).

4 Program lacks ways to acknowledge mentors' significant contributions.

\section{TRAINING:}

4 Program lacks extensive and ongoing training for working with high-needs youth.

4 Program lacks mechanisms to emphasize and foster long-term mentor involvement.

4 Program does not offer regular opportunities for mentors to gather, share moral support and resolve match issues.

FUNDING: Program lacks a clear funding mechanism to sustain the paid component of the program. 


\section{Final Thoughts}

As the mentoring field continues to grow and diversify, funders are reminded that no one program can achieve all goals for all youth. Funders need to think very carefully about whom they want to serve, what they hope to achieve and what resources they are prepared to invest. Mentoring has a wide research base to draw on, and when the right approach is selected and supported, mentoring programs can yield powerful effects for young people. Yet, there is still much to learn, particularly about the newer types of mentoring. We urge funders to invest in continued research - to determine not only whether these approaches work, but also how, for whom, and under what circumstances. This knowledge will help programs evolve in ways that are most likely to benefit youth and provide an even stronger foundation for funders' decisions about which programs to support.

\section{Endnotes}

1. This guide was made possible by generous support from The Pinkerton Foundation. Laura Johnson was an invaluable partner throughout the development of the guide and was instrumental in honing its focus, format and final direction and shepherding it through publication. Chelsea Farley did an excellent job of editing, structuring and streamlining the guide. W. Wilson Goode, Sr., Jean Grossman, Keoki Hansen, Kent Mitchell, Nadya K. Shmavonian and Judy Stavisky reviewed drafts and provided helpful feedback that shaped the final text. Malish and Pagonis developed the design.

2. A theory of change is a depiction of the changes a program expects to yield, typically in the individuals it serves, but also potentially in the broader community, public behavior, policies, etc., and how these changes are expected to come about.

3. MENTOR/National Mentoring Partnership. 2009. Elements of Effective Practice for Mentoring (3rd ed.). Alexandria, VA: MENTOR/National Mentoring Partnership. Retrieved 6/12/12 from http://www.mentoring.org/downloads/mentoring_1222.pdf.

4. "Paid mentoring" refers to programs whose main goal is to match mentors with individual youth-as opposed to programs in which paid staff work with groups of youth, potentially developing mentoring relationships with them, as a part of broader sets of services.

\section{PV V Public/Private Ventures}

